\title{
Originales
}

\section{Utilización de la ventilación no invasiva en la insuficiencia respiratoria aguda. Estudio multicéntrico en unidades de cuidados intensivos}

\author{
MIGUEL FERNÁNDEZ-VIVAS ${ }^{a}$, GUMERSINDO GONZÁLEZ-DÍAZ ${ }^{\mathrm{b}}$, JUAN CATURLA-SUCH ${ }^{\mathrm{c}}$, \\ FRANCISCO JOSÉ DELGADO-VÍLCHEZd, JOSÉ MANUEL SERRANO-SIMÓNe, \\ ANDRÉS CARRILLO-ALCARAZ ${ }^{\mathrm{b}}$, JOSÉ VAYÁ-MOSCARDÓ, JOSÉ GALCERÁ-TOMÁSa, \\ FRANCISCO ÁNGEL JAIME-SÁNCHEZ ${ }^{\circ}$ Y MANUEL SOLERA-SUÁREZ \\ aUnidad de Cuidados Intensivos. Hospital Virgen de la Arrixaca. Murcia. España. \\ bUnidad de Cuidados Intensivos. Hospital General Morales Meseguer. Murcia. España. \\ 'Unidad de Cuidados Intensivos. Hospital General de Alicante. Alicante. España. \\ dUnidad de Cuidados Intensivos. Hospital La Inmaculada. Huércal-Overa. Almería. España. \\ eUnidad de Cuidados Intensivos. Hospital Reina Sofía. Córdoba. España. \\ fUnidad de Cuidados Intensivos. Hospital Marina Baixa. Villajoyosa. Alicante. España.
}

Objetivo. Estudiar el uso de la ventilación no invasiva (VNI) en la insuficiencia respiratoria aguda, en las unidades de cuidados intensivos (UCl) de España.

Métodos. Se realizó una encuesta a $254 \mathrm{UCl}$. Al final de ésta se invitó a participar en un estudio multicéntrico retrospectivo, y proporcionar información detallada sobre pacientes ventilados.

Resultados. Contestaron 123 UCl; 119 utilizaban la VNI, de manera muy variable. En la enfermedad pulmonar obstructiva crónica (EPOC), la VNI fue de primera elección en el $89 \%$ de las unidades; en el edema agudo de pulmón (EAP), el 79\%; en la insuficiencia respiratoria tras la extubación, el $53 \%$; en la neumonía, el $53 \%$, y en el síndrome de distrés respiratorio agudo (SDRA), el 17\%. Su utilización en la EPOC fue ocasional o nula en el $11 \%$ de las unidades, $y$ en el EAP, en el $21 \%$. 18 hospitales enviaron información de 432 pacientes ventilados, de

Este estudio fue financiado parcialmente por el Instituto de Salud Carlos III, Fondo de Investigación Sanitaria (FIS) PI 04/90138; Plan Nacional de Investigación Científica, Desarrollo e Innovación Tecnológica.

Correspondencia: Dr. M. Fernández-Vivas.

Unidad de Cuidados Intensivos. Hospital Universitario Virgen de la Arrixaca.

Ctra. Madrid-Cartagena, s/n. 30120 El Palmar. Murcia. España.

Correo electrónico: mfvivas@ ono.com

Manuscrito aceptado el 27-10-2008. los que $232(54 \%)$ recibieron VNI como primera elección. La neumonía o el SDRA fueron factores independientes en relación con el fracaso de la VNI (odds ratio ajustada [ORa] = 5,71; intervalo de confianza [IC] del 95\%, 1,83-17,8; $p=0,003)$. La admisión en una unidad que ventilara de forma no invasiva a más de 50 pacientes/año (ORa $=0,22$; IC del $95 \%, 0,07-0,63 ; \mathrm{p}=0,005)$ y una mayor razón $\mathrm{PaO}_{2} /$ $\mathrm{FIO}_{2}$ tras una hora de ventilación (ORa $=0,98$ por punto; IC del 95\%, 0,97-0,99; $p<0,001$ ) fueron factores protectores.

Conclusiones. La VNI es ampliamente utilizada en las $\mathrm{UCl}$ de España, pero es posible que siga estando infrautilizada en la EPOC y el EAP. EI diagnóstico de neumonía o SDRA fue un factor independiente en relación con el fracaso. Ventilar a más de 50 pacientes/año y una mayor $\mathrm{PaO}_{2} / \mathrm{FIO}_{2}$ tras una hora fueron factores protectores.

PALABRAS CLAVE: Insuficiencia respiratoria. Estudio epidemiológico. Unidad de cuidados intensivos. Ventilación mecánica. Ventilación no invasiva.

USE OF NON-INVASIVE VENTILATION IN ACUTE RESPIRATORY FAILURE. MULTICENTER STUDY IN INTENSIVE CARE UNITS

Objectives. Study the use of non-invasive ventilation (NIV) in patients with acute respiratory failure in intensive care units (ICUs) in Spain. 
Methods. A questionnaire was sent to 254 ICUs, after which, they were invited to participate in a multicenter, retrospective study, providing detailed information on ventilated patients.

Results. Answers were received from 123 hospitals. Of these, 119 used NIV, although its use varied greatly. NIV is the treatment of choice in $89 \%$ of the units for chronic obstructive pulmonary disease (COPD), in $79 \%$ for acute pulmonary edema (APE), in $53 \%$ for postextubation failure, in $53 \%$ for pneumonia $53 \%$, and in $17 \%$ for acute respiratory distress syndrome (ARDS). It was used occasionally in COPD in $11 \%$ of the units, and in $21 \%$ of the units for APE. Eighteen hospitals provided additional information on 432 ventilated patients, 232 (54\%) of whom received NIV as first line therapy. Presence of pneumonia or acute respiratory distress syndrome (ARDS) was an independent predictive factor of NIV failure (ORa $=5.71 ; \mathrm{Cl} 95 \%, 1.83-17.8 ; \mathrm{p}=$ $0.003)$. Admission in a unit with experience in NIV in $>50$ patients/year (ORa = 0.22; Cl 95\%, 0.07-0.63; p $=0.005)$ and a higher $\mathrm{PaO}_{2} / \mathrm{FiO}_{2}$ ratio after one hour of ventilation (ORa $=0.98$ per point; $\mathrm{Cl} 95 \%, 0.97-$ $0.99 ; \mathrm{p}<0.001$ ) were protector factors.

Conclusions. In Spain, NIV is widely used but it may continue to be underused in COPD and APE. The diagnosis of pneumonia or ARDS was an independent predictive risk factor. Admission in an ICU with NIV in more than $\mathbf{5 0}$ patients/year also have higher $\mathrm{PaO}_{2} / \mathrm{FiO}_{2}$ ratio after one hour of ventilation were predictive factors of success.

KEY WORDS: Acute respiratory failure. Epidemiologic study. Intensive care unit. Mechanical ventilation. Multicenter study. Non-invasive ventilation.

\section{INTRODUCCIÓN}

Varios estudios aleatorizados ${ }^{1-6}$ demuestran que la asistencia ventilatoria no invasiva en pacientes con insuficiencia respiratoria aguda reduce la necesidad de intubación endotraqueal.

A pesar de sus evidencias favorables, la ventilación no invasiva (VNI) puede estar infrautilizada. Un estudio realizado en el Reino Unido ${ }^{7}$ reflejó que el $52 \%$ de las unidades de críticos no tenían capacidad para proporcionar VNI, y que el $68 \%$ de las unidades que la utilizaron trataron a menos de 20 pacientes por año. Carlucci et $\mathrm{al}^{9}$, en un estudio multicéntrico europeo en 42 unidades de críticos, concluyeron que sólo se aplicó VNI como primera línea de tratamiento en el 16\% de los pacientes. En un estudio en unidades de cuidados intensivos (UCI) de España en 1998, la VNI se aplicó sólo en el $4 \%$ de los pacientes ventilados ${ }^{8}$.

Poca información hay disponible sobre el uso actual de la VNI en España, por lo que llevamos a cabo el presente estudio para valorar su utilización, difusión y variabilidad.

\section{MATERIAL Y MÉTODOS}

En abril de 2005, se envió por carta un cuestionario a 254 UCI de toda España para que lo contestara un facultativo con especial interés en procesos respiratorios y ventilación mecánica. En caso de no recibir respuesta por correo, se intentó recoger la información mediante conversación telefónica o correo electrónico.

Se recogieron los siguientes datos: características de cada centro, que incluía si era universitario, si tenía docencia posgrado y el número de camas de la unidad; cómo consideraban su formación en VNI; cómo consideraban la necesidad de formación en VNI; aplicación de VNI, y en caso de no hacerlo, cuál era la razón; si había protocolo; tipo de ventilador o ventiladores utilizados; años de experiencia; número de pacientes que recibían VNI al año; frecuencia de utilización de la VNI en enfermedad obstructiva crónica (EPOC), asma, enfermedad restrictiva crónica, edema agudo de pulmón (EAP), enfermedad neuromuscular, insuficiencia tras la extubación, neumonía y síndrome de distrés respiratorio agudo (SDRA); modalidad ventilatoria más usada; utilización de tecnologías poco habituales como VNI con helio y asistencia proporcional.

Finalmente, invitábamos a dar información detallada retrospectiva de todos los pacientes ventilados de modo convencional o no invasivo entre enero y marzo de 2005. Los criterios de inclusión fueron: ser mayor de 18 años, haber tenido insuficiencia respiratoria de cualquier causa, asistida con ventilación mecánica convencional o no invasiva iniciada durante el período señalado. Los criterios de exclusión fueron: necesidad de intubación inmediata por gasping o parada cardiorrespiratoria, coma estructural (ictus, traumatismo craneoencefálico, etc.) o metabólico no causado por agudización de EPOC, shock, intubación en la UCI para un procedimiento quirúrgico y pacientes intubados en quirófano e ingresados posteriormente en UCI para control posquirúrgico.

Se registraron las siguientes variables epidemiológicas, fisiológicas y de morbimortalidad: sexo, edad, APACHE II, enfermedad subyacente, tipo de ventilación (no invasiva o convencional), modalidad ventilatoria inicial (control de volumen, presión de soporte, etc.), duración de la ventilación, variables fisiológicas respiratorias y hemodinámicas basales y a la hora de ventilación (frecuencia respiratoria, $\mathrm{pH}, \mathrm{PaCO}_{2}$, $\mathrm{CO}_{3} \mathrm{H}_{2}, \mathrm{PaO}_{2} / \mathrm{FiO}_{2}, \mathrm{SatO}_{2}$, frecuencia cardíaca y presión arterial sistólica, diastólica y media), necesidad de intubación endotraqueal si se estaba recibiendo VNI, incidencias de neumonía en relación con ventilación mecánica y traqueostomía, duración de las estancias en UCI y hospital, y mortalidad en UCI y hospitalaria.

El fracaso de la VNI se definió como la necesidad de intubación endotraqueal y/o mortalidad en UCI u hospitalaria, y el éxito, como supervivencia hospitalaria sin necesidad de intubación endotraqueal.

Para el análisis estadístico utilizamos, para las variables cualitativas, la prueba de la $\chi^{2}$ o el test de Fisher, en función de los valores esperados. Para las variables cuantitativas, según su distribución, utilizamos el test de Student o la U de Mann-Whitney. Consideramos significación estadística una $\mathrm{p}<0,05$. Realizamos un análisis de regresión logística para de- 
terminar los predictores independientes del fracaso de la VNI en el que se incluyeron las variables que en el análisis univariable fueron significativas o presentaban tendencia a serlo $(p<0,1)$. Como software estadístico empleamos el programa SPSS 11.0.

\section{RESULTADOS}

De los 254 hospitales con UCI a los que se remitió el cuestionario, 62 respondieron por escrito. Otros 61 hospitales contestaron mediante llamada telefónica, lo que hizo que el total de hospitales participantes fuese de 123 (48\%). Entre estos centros, 50 (41\%) eran universitarios y $85(69 \%)$ tenían docencia de posgrado.

Durante el período de estudio, la VNI era utilizada en 119 de los 123 hospitales participantes (97\%) y no lo era en 4 (3\%). Las causas de no utilizarla fueron: insuficiente experiencia, falta de ventilador específico para VNI, unidad recién abierta y falta de pacientes que cumplieran criterios de utilización de VNI. En ningún caso se contestó que no se utilizara por dudas sobre su beneficio.

Del total de los centros, 75 (62\%) consideraron que la formación en VNI es imprescindible; 27 (22\%), muy importante; 20 (16\%), importante, y ninguno la consideró poco necesaria o innecesaria.

La figura 1 muestra la variabilidad en el número de pacientes ventilados por año. Ventilaban menos de 10 pacientes/año el 7\% de los hospitales; de 10 a 30, el $41 \%$; de 31 a 50 , el $25 \%$; de 51 a 75 , el $15 \%$, y más de 75 , el $12 \%$.

El 8\% consideró excelente su experiencia en VNI; el 50\%, buena; el 39\%, normal; el 2\%, mala, y un 1\%, muy mala. No hubo diferencias en la experiencia en VNI en función del número de camas mayor o menor de 15 (el 59\% con experiencia excelente o buena frente al $58 \% ; \mathrm{p}=1)$. Sin embargo, los centros que manifestaron tener experiencia excelente o buena llevaban más años aplicándola $(7,5 \pm 3$ frente a $5 \pm 3 ; p$ $<0,001)$ y hubo mayor tendencia a calificar la experiencia como buena o excelente en las unidades que ventilaban a más de 50 pacientes/año (el 69 frente al $56 \% ; \mathrm{p}=0,2)$.

Había protocolo de utilización de VNI en 45 (38,5\%) hospitales; 70 (59\%) UCI utilizaban sólo ventiladores con software específico para VNI, 19 (16\%) utilizaban sólo ventiladores clásicos de UCI sin software específico y 30 (25\%), ambos. Los hospitales con mayor experiencia utilizaban más frecuentemente VNI con software específico (el 63 frente al $36 \% ; \mathrm{p}=0,029)$.

En caso de insuficiencia respiratoria hipercápnica asistida con VNI, la modalidad ventilatoria más usada fue la presión de soporte $(75 \%)$, seguida por la presión positiva continua en la vía aérea (CPAP) en el $15 \%$, control de volumen en el $5 \%$ y control de presión en el 4\%. En la insuficiencia respiratoria hipoxémica se utilizó presión de soporte en un 53\%, CPAP en el $31 \%$, control de volumen en el $10 \%$ y control de presión en el $5 \%$ (fig. 2). Fue infrecuente la utilización de helio y asistencia proporcional; 106 (89\%)

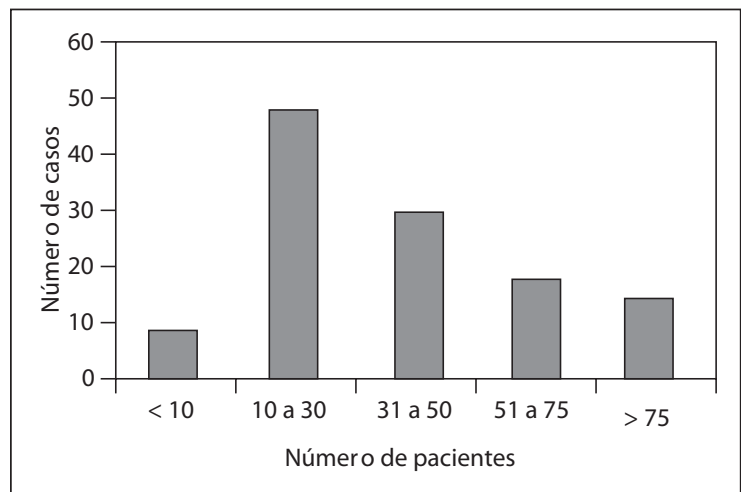

Figura 1. Número de pacientes con ventilación no invasiva por año.

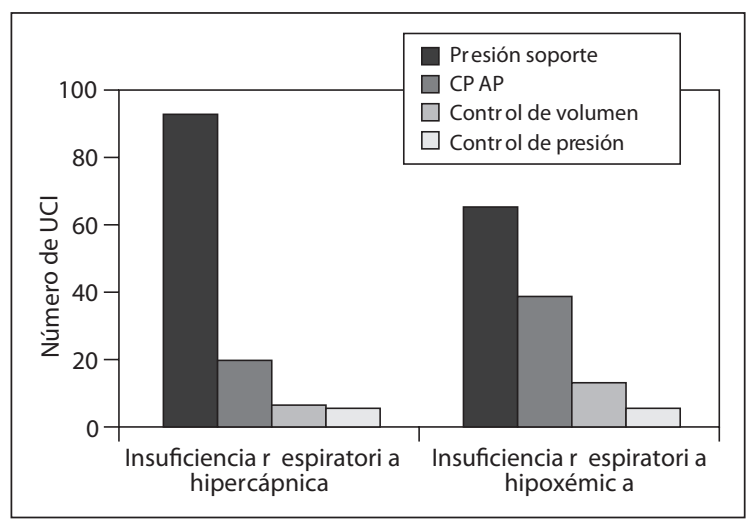

Figura 2. Método ventilatorio utilizado. CPAP: presión positiva continua en la vía aérea.

unidades jamás utilizaron helio y 99 (84\%) nunca usaron asistencia proporcional.

En la figura 3 se contempla que la utilización de la VNI, como método inicial en caso de insuficiencia respiratoria aguda, fue muy variable en función de la enfermedad. En pacientes con EPOC, la VNI se usó siempre, o al menos frecuentemente, como primera elección en el 89\% de las unidades; en EAP, en el 79\%; en insuficiencia respiratoria tras extubación, en el 53\%; en neumonía, en el 53\%, y en SDRA, en el $17 \%$.

De los 123 hospitales que respondieron, 18 (15\%) proporcionaron información adicional sobre pacientes; 432 pacientes cumplieron los criterios de inclusión. Inicialmente se optó por la VNI en 232 (54\%) pacientes. Algunas peculiaridades clínicas en relación con la instauración de la VNI fueron: mayor edad, tendencia a menor porcentaje de varones, menor puntuación del APACHE II, menor frecuencia cardíaca y mayor presión arterial media. Respecto a los parámetros de función respiratoria, la VNI se asoció con una mayor frecuencia respiratoria y un mayor valor de hipercapnia, en presencia de una similar relación $\mathrm{PaO}_{2} / \mathrm{FiO}_{2}$ y pH en la gasometría inicial (tabla 1). Su utilización fue más frecuente en la insuficiencia respiratoria hipercápnica (el 44 frente al 32\%; $\mathrm{p}<0,01)$ y menor en la hipóxemica (el 56 frente al 


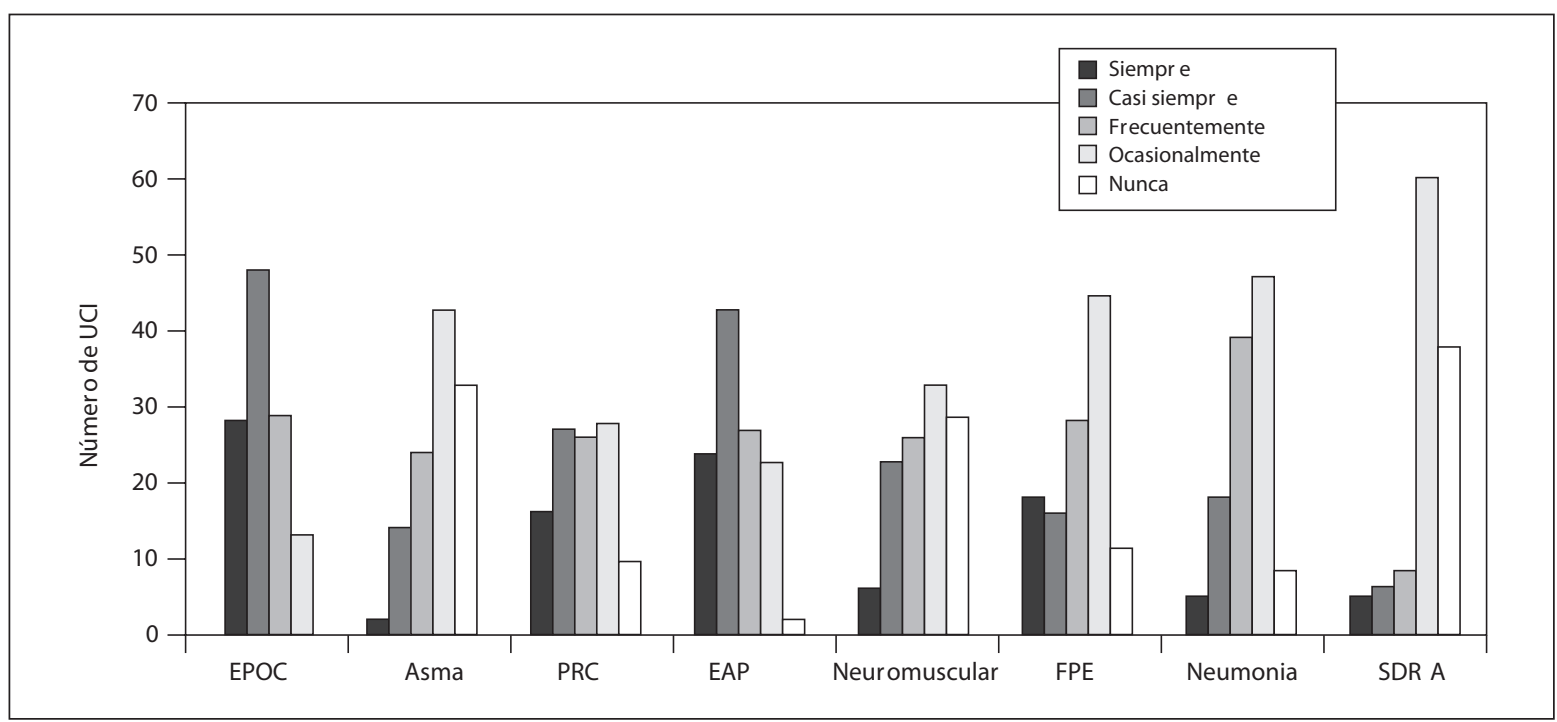

Figura 3. Utilización de la ventilación no invasiva (VNI) en función de la enfermedad. EAP: edema agudo de pulmón; EPOC: enfermedad pulmonar obstructiva crónica; FPE: fracaso postextubación; PRC: enfermedad restrictiva crónica; SDRA: síndrome de distrés respiratorio agudo.

$68 \%$; $<<0,01)$. La proporción de los pacientes con EPOC fue mayor en el grupo de VNI (el 30 frente al $17 \%$; $<$ 0,01) y menor la de aquellos con neumonía (el 10 frente al 30\%; p < 0,01).

\section{TABLA 1. Características basales de los pacientes}

\begin{tabular}{|c|c|c|c|}
\hline & $\mathrm{VNI}(\mathrm{n}=232)$ & $\operatorname{VMC}(\mathrm{n}=200)$ & $\mathrm{p}$ \\
\hline Varones & $139(60 \%)$ & $136(68 \%)$ & 0,08 \\
\hline Edad (años) & $67 \pm 14$ & $63 \pm 16$ & $<0,01$ \\
\hline APACHE II & $18 \pm 7$ & $21 \pm 8$ & $<0,001$ \\
\hline $\begin{array}{l}\text { Insuficiencia respiratora } \\
\text { hipercápnica }\end{array}$ & $101(44 \%)$ & $63(32 \%)$ & 0,01 \\
\hline EPOC & $69(30 \%)$ & $34(17 \%)$ & $<0,01$ \\
\hline $\begin{array}{l}\text { Enfermedad } \\
\text { restrictiva crónica }\end{array}$ & $14(6 \%)$ & $5(2,5 \%)$ & 0,12 \\
\hline $\begin{array}{l}\text { Enfermedad } \\
\text { neuromuscular }\end{array}$ & $0(0 \%)$ & $10(5 \%)$ & $<0,01$ \\
\hline $\begin{array}{l}\text { Insuficiencia } \\
\text { hipercápnica } \\
\text { tras extubación }\end{array}$ & $11(5 \%)$ & $3(1,5 \%)$ & 0,1 \\
\hline Otros & $7(3 \%)$ & $11(6 \%)$ & 0,3 \\
\hline $\begin{array}{l}\text { Insuficiencia } \\
\text { respiratoria } \\
\text { hipoxémica }\end{array}$ & $131(56 \%)$ & $137(68 \%)$ & 0,01 \\
\hline EAP & $60(26 \%)$ & $41(21 \%)$ & 0,23 \\
\hline Neumonía & $24(10 \%)$ & $60(30 \%)$ & $<0,001$ \\
\hline SDRA & $18(8 \%)$ & $20(10 \%)$ & 0,52 \\
\hline Traumatismo torácico & $6(2 \%)$ & $6(3 \%)$ & 0,97 \\
\hline \multicolumn{4}{|l|}{ Insuficiencia } \\
\hline $\begin{array}{l}\text { hipoxémica tras } \\
\text { extubación }\end{array}$ & $18(8 \%)$ & $5(2 \%)$ & 0,03 \\
\hline Otros & $5(2 \%)$ & $5(2 \%)$ & 0,93 \\
\hline FR (respiraciones/min) & $33 \pm 8$ & $31 \pm 9$ & 0,04 \\
\hline $\mathrm{pH}$ & $7,28 \pm 0,12$ & $7,28 \pm 0,13$ & 0,51 \\
\hline $\mathrm{PaCO} 2(\mathrm{mmHg})$ & $64 \pm 27$ & $56 \pm 26$ & $<0,01$ \\
\hline $\mathrm{CO} 3 \mathrm{H}(\mathrm{mmol} / \mathrm{l})$ & $28 \pm 8$ & $24 \pm 8$ & $<0,001$ \\
\hline $\mathrm{PaO} 2 / \mathrm{FiO} 2$ & $151 \pm 70$ & $147 \pm 84$ & 0,60 \\
\hline FC (lat/min) & $104 \pm 21$ & $110 \pm 27$ & 0,03 \\
\hline PAM (mmHg) & $93 \pm 23$ & $85 \pm 26$ & $<0,01$ \\
\hline
\end{tabular}

EAP: edema agudo de pulmón; EPOC: enfermedad pulmonar obstructiva crónica; FC: frecuencia cardíaca; FR: frecuencia respiratoria; PAM: presión arterial media; SDRA síndrome disneico agudo; VMC: ventilación mecánica convencional; VNI: ventilación no invasiva.
Entre los pacientes que recibieron VNI, 55 (24\%) requirieron intubación endotraqueal, y las incidencias de mortalidad en UCI y hospitalaria fueron del 18 y el $24 \%$, respectivamente. En la tabla 2 se presentan estos y otros resultados tanto generales como en los subgrupos de insuficiencia respiratoria hipercápnica e hipoxémica, y en los principales procesos patológicos.

La VNI conllevó éxito en 142 de 232 pacientes (61\%). En la tabla 3 mostramos las principales características de la población en VNI en función del éxito o el fracaso. El éxito de la VNI se acompañó de menor duración de la ventilación $(3 \pm 3$ frente a $11 \pm 13$; $\mathrm{p}<0,001)$, estancia en UCI $(5 \pm 4$ frente a $14 \pm 15 ; \mathrm{p}$ $<0,001)$, estancia hospitalaria $(18 \pm 10$ frente a $22 \pm$ $19 ; \mathrm{p}=0,04)$ e incidencia de neumonía en relación con ventilación mecánica (el 0 frente al 4\%; p = 0,02).

La incidencia de fracaso de la VNI fue menor (el 35 frente al $58 \% ; p=0,012$ ) entre los pacientes ingresados en unidades que realizaban ventilación no invasiva a más de 50 pacientes al año.

Los pacientes con fracaso de la VNI tenían mayor APACHE II (20 \pm 7 frente a $17 \pm 6 ; p=0,003)$ y más diagnósticos de neumonía o SDRA (el 30 frente al $11 \% ; \mathrm{p}<0,001)$, y menor presión arterial basal media $(89 \pm 18$ frente a $96 \pm 24 ; \mathrm{p}=0,03)$ y menos diagnósticos de EPOC (el 41 frente al 65\%; p < 0,001). A la hora de ventilación, el grupo de fracaso tenía mayor frecuencia respiratoria $(28 \pm 8$ frente a $25 \pm 6 ; \mathrm{p}=$ $0,003)$, menor $\mathrm{pH}(7,31 \pm 0,09$ frente a 7,34 $\pm 0,08 ; \mathrm{p}$ $=0,04)$, menor razón $\mathrm{PaO}_{2} / \mathrm{FiO}_{2}(148 \pm 80$ frente a $191 \pm 63 ; \mathrm{p}<0,001)$ y tendencia a menor presión arterial media $(87 \pm 16$ frente a $91 \pm 16 ; p=0,07)$.

El análisis multivariable identificó la presencia de neumonía o SDRA como factor independientemente asociado al fracaso de la VNI (odds ratio ajustada $[\mathrm{ORa}]=5,71$; intervalo de confianza [IC] del 95\%, 
TABLA 2. Respuesta a la ventilación no invasiva

\begin{tabular}{|l|c|c|c|c|c|c|c|}
\hline & $\begin{array}{c}\text { Total } \\
(\mathrm{n}=232)\end{array}$ & $\begin{array}{c}\text { IR hipercápnica } \\
(\mathrm{n}=101)\end{array}$ & $\begin{array}{c}\text { IR hipoxémica } \\
(\mathrm{n}=131)\end{array}$ & $\begin{array}{c}\text { EPOC } \\
(\mathrm{n}=69)\end{array}$ & $\begin{array}{c}\text { EAP } \\
(\mathrm{n}=60)\end{array}$ & $\begin{array}{c}\text { Neumonía } \\
(\mathrm{n}=24)\end{array}$ & $\begin{array}{c}\text { SDRA } \\
(\mathrm{n}=18)\end{array}$ \\
\hline NAV & $6(3 \%)$ & $4(4 \%)$ & $2(2 \%)$ & $1(1 \%)$ & $1(2 \%)$ & 0 & 0 \\
Traqueostomía & $10(4 \%)$ & $2(2 \%)$ & $8(6 \%)$ & $2(3 \%)$ & $2(3 \%)$ & $3(12 \%)$ & 0 \\
Días en ventilación & $6 \pm 9$ & $3 \pm 2$ & $7 \pm 11$ & $5 \pm 7$ & $5 \pm 10$ & $7 \pm 7$ & $8 \pm 8$ \\
IET tras VNI & $55(24 \%)$ & $16(16 \%)$ & $40(31 \%)$ & $10(14 \%)$ & $9(15 \%)$ & $12(50 \%)$ & $9(50 \%)$ \\
Estancia en UCI (días) & $8 \pm 10$ & $6 \pm 8$ & $10 \pm 12$ & $6 \pm 8$ & $7 \pm 10$ & $11 \pm 10$ & $11 \pm 9$ \\
Estancia hospitalaria (días) & $20 \pm 14$ & $17 \pm 12$ & $22 \pm 16$ & $17 \pm 13$ & $18 \pm 12$ & $17 \pm 13$ & $25 \pm 15$ \\
Mortalidad en UCI & $41(18 \%)$ & $11(11 \%)$ & $30(23 \%)$ & $4(6 \%)$ & $11(18 \%)$ & $9(37 \%)$ & $7(39 \%)$ \\
Mortalidad hospitalaria & $65(28 \%)$ & $26(26 \%)$ & $39(30 \%)$ & $12(17 \%)$ & $18(30 \%)$ & $10(42 \%)$ & $8(45 \%)$ \\
\hline
\end{tabular}

EAP: edema agudo de pulmón; EPOC: enfermedad pulmonar obstructiva crónica; IET: intubación endotraqueal; IR: insuficiencia respiratoria; NAV: neumonía asociada a ventilación mecánica; SDRA: síndrome de distrés respiratorio agudo; UCI: unidad de cuidados intensivos.

1,83-17,8; $\mathrm{p}=0,003)$. La admisión en una unidad que ventilara no invasivamente a más de 50 pacientes/año $(\mathrm{ORa}=0,22 ; \mathrm{IC}$ del 95\%, 0,07-0,63; $\mathrm{p}=0,005)$ y una mayor razón $\mathrm{PaO}_{2} / \mathrm{FIO}_{2}$ tras $1 \mathrm{~h}$ de ventilación (ORa $=0,98$ por punto; IC del $95 \%, 0,97-0,99 ; \mathrm{p}<0,001)$ fueron factores protectores. El valor del APACHE II mostró una OR de 1,07 por punto (IC del 95\%, 0,998$1,14 ; \mathrm{p}=0,057)$.

Para determinar si el fracaso de la VNI con necesidad de intubación suponía mayor riesgo que la intubación inicial, comparamos los 55 pacientes que precisaron intubación tras un período de VNI con los 200 intubados inicialmente. No encontramos diferencias significativas en la incidencia de neumonía en relación con ventilación mecánica (el 16 frente al 18\%; $\mathrm{p}=0,72$ ), traqueostomía (el 18 frente al 15\%; $\mathrm{p}=$ 0,51 ), mortalidad en UCI (el 47 frente al 38\%; $\mathrm{p}=$ $0,21)$ ni en la estancia hospitalaria $(28 \pm 21$ frente a $26 \pm 27 ; p=0,58)$, pero hubo tendencias a mayor estancia en UCI $(19 \pm 16$ frente a $14 \pm 17$; $\mathrm{p}=0,051)$ y mayor mortalidad hospitalaria (el 55 frente al 43\%; $\mathrm{p}=0,13$ ) en el grupo de fracaso de VNI.

\section{DISCUSIÓN}

Ateniéndonos a los 123 centros que contestaron a nuestra encuesta, consideramos que la utilización de la VNI en España es relativamente frecuente, y que la respuesta puede reflejar el interés que despierta la VNI como una técnica atractiva y útil.

En un estudio ${ }^{7}$ llevado a cabo en el Reino Unido en 1997 sólo el $48 \%$ de los hospitales participantes tenían disponibilidad de VNI, lo cual contrasta con su empleo en el $97 \%$ de nuestros centros participantes. Este hallazgo puede ser consecuencia de la diferencia temporal entre la obtención de los datos en ambos estudios, de modo que estudios epidemiológicos más recientes evidencian mayores difusión y frecuencia de aplicación de la VNI, del $98 \%{ }^{10}$ y el $100 \%{ }^{11}$.

Algunas causas de la mayor utilización de la VNI pueden ser interpretadas con los datos obtenidos hace 10 años por Doherty et al $^{7}$. Según ellos, el $15 \%$ de los hospitales que no empleaban VNI lo hacían por dudas sobre su beneficio. En nuestro caso, y en concordancia con Maheshwari et $\mathrm{al}^{10}$, en ningún caso se justificó no aplicar la VNI por dudas sobre su beneficio. El hecho de que un $84 \%$ contestara que la formación en
TABLA 3. Características basales y resultados según el éxito o el fracaso de la ventilación no invasiva (VNI)

\begin{tabular}{|c|c|c|c|}
\hline & $\begin{array}{l}\text { Fracaso de } \\
\text { VNI }(n=90)\end{array}$ & $\begin{array}{c}\text { Éxito de } \\
\text { VNI }(\mathrm{n}=142)\end{array}$ & $\mathrm{p}$ \\
\hline Varones & $55(61 \%)$ & $84(59 \%)$ & 0,77 \\
\hline Edad (años) & $68 \pm 14$ & $66 \pm 14$ & 0,32 \\
\hline APACHE II & $20 \pm 7$ & $17 \pm 6$ & 0,003 \\
\hline $\begin{array}{l}\text { Insuficiencia respiratoria } \\
\text { hipoxémica }\end{array}$ & $57(63 \%)$ & $74(52 \%)$ & 0,09 \\
\hline Neumonía o SDRA & $27(30 \%)$ & $15(11 \%)$ & $<0,001$ \\
\hline EPOC о EAP & $37(41 \%)$ & $92(65 \%)$ & $<0,001$ \\
\hline \multicolumn{4}{|l|}{$\begin{array}{l}\text { Variables fisiológicas } \\
\text { basales }\end{array}$} \\
\hline FR (respiraciones/min) & $32 \pm 9$ & $33 \pm 8$ & 0,87 \\
\hline $\mathrm{pH}$ & $7,28 \pm 0,11$ & $7,29 \pm 0,12$ & 0,54 \\
\hline $\mathrm{PaCO}_{2}(\mathrm{mmHg})$ & $62 \pm 26$ & $65 \pm 29$ & 0,42 \\
\hline $\mathrm{CO}_{3} \mathrm{H}(\mathrm{mmol} / \mathrm{l})$ & $27 \pm 7$ & $28 \pm 8$ & 0,13 \\
\hline $\mathrm{PaO}_{2} / \mathrm{FiO}_{2}$ & $141 \pm 78$ & $156 \pm 65$ & 0,13 \\
\hline FC (lat/min) & $104 \pm 23$ & $104 \pm 21$ & 0,89 \\
\hline PAM (mmHg) & $89 \pm 18$ & $96 \pm 24$ & 0,03 \\
\hline \multicolumn{4}{|l|}{$\begin{array}{l}\text { Variables fisiológicas } \\
\text { a la hora } \\
\text { de ventilación }\end{array}$} \\
\hline $\begin{array}{l}\text { FR (respiraciones/ } \\
\text { min) }\end{array}$ & $28 \pm 8$ & $25 \pm 6$ & 0,003 \\
\hline $\mathrm{pH}$ & $7,31 \pm 0,09$ & $7,34 \pm 0,08$ & 0,04 \\
\hline $\mathrm{PaCO}_{2}(\mathrm{mmHg})$ & $58 \pm 24$ & $56 \pm 20$ & 0,57 \\
\hline $\mathrm{CO}_{3} \mathrm{H}(\mathrm{mmHg})$ & $28 \pm 8$ & $29 \pm 7$ & 0,19 \\
\hline $\mathrm{PaO}_{2} / \mathrm{FiO}_{2}$ & $148 \pm 80$ & $191 \pm 63$ & $<0,001$ \\
\hline FC (lat/min) & $100 \pm 22$ & $97 \pm 19$ & 0,33 \\
\hline PAM (mmHg) & $87 \pm 16$ & $91 \pm 16$ & 0,07 \\
\hline
\end{tabular}

EAP: edema agudo de pulmón; EPOC: enfermedad pulmonar obstructiva crónica; $\mathrm{FC}$ : frecuencia cardíaca; FR: frecuencia respiratoria; PAM: presión arterial media; SDRA: síndrome de distrés respiratorio agudo.

VNI es muy importante o imprescindible; un 16\%, importante, y en ningún caso se considerara poco necesaria o innecesaria, es otra muestra de la trascendencia que ha adquirido esta técnica en nuestras UCI.

El aumento del número de unidades que aplican VNI va acompañado de un incremento en el número de pacientes que la reciben. En el estudio de Doherty et $\mathrm{al}^{7}$ en 1997 , el $42 \%$ de los centros que aplicaron VNI ventilaban a menos de 10 pacientes/año y sólo un $15 \%$ de los hospitales ventilaban a más de 40 pacientes/año, mientras que en el nuestro, el 52\% ventila a más de 30 pacientes/año y el $27 \%$, a más de 50 pacientes/año. En un estudio multicéntrico francés ${ }^{9}$, con datos recogidos en 1997, se aplicó VNI inicial- 
mente sólo en el $16 \%$ del total de los pacientes y en el $35 \%$ de los pacientes que ingresaron en UCI sin intubación. Otro estudio multicéntrico francés ${ }^{11}$, con datos recogidos 5 años después, demostró un aumento significativo de la aplicación de VNI como primera elección en el total de los pacientes (el 23 frente al $16 \%$ ) y, sobre todo, en los pacientes ingresados en UCI sin intubación (el 52 frente al 35\%). En el estudio de Frutos et $\mathrm{al}^{8}$ sobre el uso de la ventilación mecánica en 72 UCI de España en 1998, la utilización de la VNI fue muy baja (4\%). En otro estudio multicéntrico, en pacientes con EPOC, sólo se utilizó VNI en el $16 \%$ de los pacientes ventilados ${ }^{12}$. En nuestro estudio, 18 hospitales enviaron información detallada de 432 pacientes, de los que el 54\% recibió VNI como primera elección. Dado que excluimos a los pacientes que venían intubados por proceder de quirófano, presentar gasping o parada respiratoria, coma estructural o metabólico, etc, la utilización de la VNI como primera línea en el 54\% de los pacientes de nuestro estudio es muy similar al $52 \%$ señalado por Demoule et $\mathrm{al}^{11}$, y confirma el importante incremento de su uso en los últimos años. Es posible que la conferencia de consenso de 1999 pueda haber influido en esta evolución ${ }^{13}$, pero también se ha indicado que los clínicos cambian su práctica habitual cuando perciben que el cambio es beneficioso para sus pacientes ${ }^{14}$. Muchos intensivistas pueden tener la impresión de que al aplicar la VNI a un paciente con EAP se consigue una mejoría más rápida del trabajo respiratorio y de la oxigenación, y que su aplicación a un paciente con EPOC puede evitar la intubación y los problemas derivados de la ventilación mecánica convencional y del destete en este tipo de pacientes.

La utilización de la VNI como primera elección fue muy variable, como ocurrió en otros estudios ${ }^{9-11}$. Los hospitales que manifestaron tener una experiencia buena o excelente en VNI llevaban más años aplicándola, con una tendencia no significativa a ventilar de modo no invasivo a más de 50 pacientes/año. Como se ha propuesto, el aprendizaje de la VNI es fundamental para su éxito ${ }^{15}$, de modo que, conforme ha aumentado su utilización, han disminuido las incidencias de neumonía nosocomial y la mortalidad.

Respecto a los ventiladores utilizados, la mayoría de los centros $(59 \%)$ utilizaron sólo ventiladores específicos de VNI, a diferencia de estudios epidemiológicos previos que utilizaron más frecuentemente ventiladores convencionales ${ }^{10,11}$. En nuestro estudio la peor experiencia percibida de la aplicación de VNI se asoció a la utilización de ventiladores sin software específico. Nuestro hallazgo guarda cierta relación con la observación de que los hospitales con menos de 3 ventiladores específicos de VNI aplican esta técnica con menor frecuencia ${ }^{10}$. Los ventiladores convencionales pueden compensar peor las fugas y proporcionar un menor confort al paciente, lo que puede contribuir tanto a una peor percepción de la utilidad de la VNI como a su menor aplicación.

Como en estudios previos, el modo ventilatorio más usado fue la presión de soporte ${ }^{9,11}$, especialmente en caso de insuficiencia respiratoria hipercápnica.
Es llamativa la gran variabilidad en el uso de la VNI en función de la enfermedad. A pesar de la evidencia favorable para la utilización de VNI en enfermedades como EPOC y EAP ${ }^{13,16}$, en la primera se utiliza de manera ocasional en el $11 \%$ de las unidades, y en el EAP, su uso es ocasional o nulo en el $21 \%$. Por el contrario, en procesos donde la utilización de la VNI suscita controversia, como la neumonía ${ }^{2,17-19}$ y la insuficiencia tras extubación ${ }^{20-25}$, el 53\% de las unidades la utilizan como elección siempre o, al menos, frecuentemente. Por último, en el SDRA la mayoría de las unidades (83\%) no utilizan la VNI o lo hacen de manera ocasional. En definitiva, aunque el empleo de la VNI ha aumentado en cantidad y calidad en los últimos años, es posible que siga estando infrautilizada en enfermedades como la EPOC y el EAP.

En los 18 hospitales que proporcionaron información adicional, algunas de las diferencias documentadas en las características de los pacientes sometidos inicialmente a VNI y los ventilados de forma invasiva pueden ser explicadas de forma plausible. Así, la ventilación mecánica convencional como primera opción fue más frecuente en los pacientes más graves, con mayor APACHE II, diagnóstico de neumonía, menor bicarbonato, menor presión arterial media y mayor frecuencia cardíaca.

Algunos estudios previos concluyeron que la efectividad de la VNI en la práctica diaria era similar a la eficacia conseguida por equipos investigadores ${ }^{21,26}$. En un estudio retrospectivo sobre la aplicación de VNI en insuficiencia respiratoria aguda de diversas causas en un hospital docente ${ }^{27}$, se evitó la intubación en el $63 \%$ de los casos. En otros 2 estudios multicéntricos prospectivos no aleatorizados, la VNI evitó la intubación en el $60 \%{ }^{9}$ y el $62 \%^{11}$ de los casos. En consonancia con los previos, nuestro estudio confirma la efectividad de la VNI en la práctica diaria, ya que evita la intubación endotraqueal en el $76 \%$ de los casos.

Considerando el éxito de la VNI como la supervivencia hospitalaria sin necesidad de intubación endotraqueal, se consiguió en el $61 \%$ de los pacientes, tasa similar a la reflejada por otros estudios ${ }^{9,11}$. El éxito de la VNI conllevó menor duración de la ventilación mecánica, menores estancias en UCI y hospitalaria y menor incidencia de neumonía en relación con la ventilación mecánica. Estudiamos las variables que podían influir en el éxito o el fracaso de la técnica mediante un análisis de regresión logística. El diagnóstico de neumonía o SDRA fue un factor predictivo independiente de fracaso, mientras que la razón $\mathrm{PaO}_{2} /$ $\mathrm{FiO}_{2}$ a la hora de ventilación y estar ingresado en una unidad que ventila de modo no invasivo a más de 50 pacientes/año fueron factores predictivos independientes de éxito. Antonelli et $\mathrm{al}^{28}$, al igual que nosotros, encontraron que la gasometría arterial basal no tuvo valor predictivo y sí lo tuvo la razón $\mathrm{PaO}_{2} / \mathrm{FiO}_{2}$ a la hora de ventilación, y también identificó el diagnóstico de neumonía o SDRA como variable predictiva independiente de fracaso de la VNI. Esto puede estar en relación con el tiempo de recuperación de la 
enfermedad subyacente, que es menor en pacientes con agudización de EPOC o EAP que en pacientes con insuficiencia respiratoria hipoxémica de otra etiología. El estudio de Domenighetti et al ${ }^{29}$ demostró esta diferencia; compararon la evolución de los pacientes con neumonía y EAP que presentaban una razón $\mathrm{PaO}_{2} / \mathrm{FiO}_{2}$ basal similar, asistidos con VNI, y observaron una menor duración de la ventilación y mayor tasa de éxito en el grupo de EAP. Un reciente estudio $^{30}$ demostró que el éxito de la VNI conllevaba menores estancias en UCI, menor riesgo de neumonía en relación con la ventilación mecánica y mayor supervivencia que en los pacientes con ventilación mecánica convencional, mientras que el fracaso de la VNI tenía un impacto diferente en la mortalidad en función de la causa de la insuficiencia respiratoria. El fracaso de la VNI conllevó mayor mortalidad en la insuficiencia respiratoria aguda de etiología diferente del EAP o agudización de la EPOC.

En nuestro estudio, estar ingresado en una unidad que ventila de modo no invasivo a más de 50 pacientes/año también fue factor predictivo protector independiente. Otro estudio ${ }^{15}$ mostró que, a lo largo de los años, la utilización de la VNI en pacientes con EPOC y EAP fue aumentando, mientras que las incidencias de neumonía nosocomial y mortalidad fueron disminuyendo, esto indica que había un efecto aprendizaje y que el aumento en la experiencia de la VNI era fundamental para su éxito.

Para determinar si el fracaso de la VNI con necesidad de intubación suponía mayor riesgo que la intubación inicial, comparamos a los 55 pacientes que precisaron intubación tras un período de VNI con los 200 que fueron intubados inicialmente, y encontramos tendencias a mayor estancia en UCI y mayor mortalidad hospitalaria en el grupo de fracaso de VNI. En el estudio de Carlucci et $\mathrm{al}^{9}$, la mala evolución con la VNI no tuvo relación con mayor mortalidad, pero sí con una duración más prolongada de la ventilación mecánica y mayor estancia en UCI. Estos hallazgos indican que una mala respuesta a la VNI conlleva peor pronóstico y, por lo tanto, el retraso de la intubación podría ser perjudicial para el paciente $\mathrm{p}^{9,24}$.

Nuestro estudio tiene algunas limitaciones. En primer lugar sólo se obtuvo respuesta de algo menos de la mitad de los hospitales a los que nos dirigimos. Es probable que este hecho sesgase la inclusión de las unidades con más interés en la utilización de la VNI. En segundo lugar, la información sobre el empleo de la VNI se limita al año 2005 y, de acuerdo con los argumentos de la discusión, es muy probable que en este intervalo de tiempo haya habido una mayor implantación de esta técnica. Las respuestas podían ser aproximaciones a la realidad ya que no siempre se disponía de datos seguros registrados, y eran dadas por un solo clínico de cada servicio; es posible que otros facultativos tuvieran opiniones diferentes. Otra limitación es la naturaleza retrospectiva de los datos de los pacientes de los centros que proporcionaron dicha información detallada. Por otra parte, el hecho de haberlo realizado exclusivamente en UCI, sin incluir a pacientes que recibieron VNI en servicios de urgencias u otras plantas hospitalarias, puede subestimar su aplicación real. En cualquier caso, creemos que nuestros resultados reflejan la actividad de un considerable número de centros $\mathrm{y}$, por lo tanto, es muy probable que puedan interpretarse como una información valiosa representativa de las peculiaridades ligadas a la utilización de la VNI en las UCI de España.

\section{Investigadores que participaron en el estudio}

J.AcostaEscribano (Hospital General Universitario de Alicante) O. Agudo Pascual (Hospital García Orcoyen de Estella, Navarra), M. Alcázar Espín (Hospital General Universitario Morales Meseguer de Murcia), J.M. Allegue Gallego (Hospital Sta. María del Rosell de Cartagena, Murcia), S. Almanza López (Hospital General Universitario de Alicante), M. Alonso Rubio (Hospital Universitario Virgen de la Arrixaca, Murcia), A. Arizmendi Demay (Hospital de Tarrasa, Barcelona), M.P. Auguiano Baquero (Hospital García Orcoyen de Estella, Navarra), A. Belenguer Muncharaz (Hospital General de Castellón), M.J. Broch Porcar (Hospital de Sagunto, Valencia), J.L. Buendía Moreno (Hospital Universitario Virgen de la Arrixaca, Murcia), J.J. Díaz Díaz (Hospital Dr. Negrín, las Palmas de Gran Canaria), A. Esquinas Rodríguez (Hospital General Universitario Morales Meseguer, Murcia), E. García Piney (Hospital de Cabueñes, Gijón, Asturias), M. Gobernado Serrano (Hospital Sta. Bárbara, Soria), E. Gómez Martínez (Hospital de Sagunto, Valencia), O. Jiménez Merino (Hospital Dr. Negrín, las Palmas de Gran Canaria), J.M. Martínez Segura (Clínica San Miguel, Pamplona, Navarra), A. Mula Gómez (Hospital Universitario Reina Sofía, Córdoba), J.F. Murcia Payá (Hospital Santa María del Rosell de Cartagena, Murcia), F.J. Olea Parejo (Hospital de Cabueñes, Gijón,Asturias), C. Pérez Calvo(Fundación Jiménez Díaz, Madrid), M. Pérez Márquez (Fundación Jiménez Díaz, Madrid), A. Quintano Rodero (Hospital Universitario Marqués de Valdecilla, Santander), F. Rodríguez Albarrán (Clínica San Miguel, Pamplona, Navarra), J.J. Rubio Muñoz (Hospital Universitario Puerta de Hierro, Madrid), J. Sánchez Payá (Hospital General Universitario de Alicante), B. Suberviola Cañas (Hospital Universitario Marqués de Valdecilla, Santander), F. Tena Espeleta (Hospital Santa Bárbara, Soria), G. Torres Martínez (Hospital Universitario Virgen de la Arrixaca, Murcia), J. Trenchs Verdaguer (Hospital de Tarrasa, Barcelona), M. Valdivia de la Fuente (Hospital Universitario Puerta de Hierro, Madrid), B. Vidal Tegedor (Hospital General de Castellón).

\section{BIBLIOGRAFÍA}

1. Brochard L, Mancebo J, Wysocki M, Lofaso F, Conti G, Rauss A, et al. Noninvasive ventilation for acute exacerbations of chronic obstructive pulmonary disease. N Engl J Med. 1995;333:817-22. 
2. Antonelli M, Conti G, Rocco M, Bufi M, De Blasi RA, Vivino $\mathrm{G}$, et al. A comparison of noninvasive positive-pressure ventilation and conventional mechanical ventilation in patients with acute respiratory failure. N Engl J Med. 1998;339:429-35.

3. Bott J, Carroll MP, Conway JH, Keilty SE, Ward EM, Brown AM, et al. Randomised controlled trial of nasal ventilation in acute ventilatory failure due to chronic obstructive airways disease. Lancet. 1993;341:1555-7.

4. Nava S, Ambrosino N, Clini E, Prato M, Orlando G, Vitacca $\mathrm{M}$, et al. Noninvasive mechanical ventilation in the weaning of patients with respiratory failure due to chronic obstructive pulmonary disease: a randomized, controlled trial. Ann Intern Med. 1998; 128:721-8.

5. Confalonieri M, Potena A, Carbone G, Porta RD, Tolley EA, Umberto Meduri G. Acute respiratory failure in patients with severe community-acquired pneumonia: a prospective randomized evaluation of non-invasive ventilation. Am J Respir Crit Care Med. 1999;160:1585-91.

6. Masip J, Betbesé AJ, Páez J, Vecilla F, Cañizares R, Padró J, et al. Non-invasive pressure support ventilation versus conventional oxygen therapy in acute cardiogenic pulmonary oedema: a randomised trial. Lancet. 2000;356:2126-32.

7. Doherty MJ, Greenstone MA. Survey of non-invasive ventilation (NIPPV) in patients with acute exacerbations of chronic obstructive pulmonary disease (COPD) in the UK. Thorax. 1998:53:863-6.

8. Frutos F, Alía I, Lorenzo MR, García Pardo J, Nolla M, Ibáñez $\mathrm{J}$, et al. Utilización de la ventilación mecánica en 72 unidades de cuidados intensivos en España. Med Intensiva. 2003;27:1-12.

9. Carlucci A, Richard JC, Wysocki M, Lepage E, Brochard L and the SRLF Collaborative Group on Mechanical Ventilation. Noninvasive versus conventional mechanical ventilation. An epidemiologic survey. Am J Respir Crit Care Med. 2001;163:874-80.

10. Maheshwari V, Paioli D, Rothaar R, Hill NS. Utilization of noninvasive ventilation in acute care hospitals. Chest. 2006;129:1226-33.

11. Demolue A, Girou E, Richard JC, Taillé S, Brochard L. Increased use of noninvasive ventilation in French intensive care units. Intensive Care Med. 2006;32:1747-55.

12. Frutos Vivar F, Esteban A, Anzueto A, Apezteguía C, González M, Bugedo G. Pronóstico de los enfermos con enfermedad pulmonar obstructiva crónica que precisan ventilación mecánica. Med Intensiva. 2006;30:52-61.

13. Evans TW. International Consensus Conferences in Intensive Care Medicine: Non-invasive positive pressure ventilation in acute respiratory failure. Intensive Care Med. 2001;27:166-78.

14. Greco PJ, Eisenberg JM. Changing physicians' practices. N Engl J Med. 1993;329:1271-3.

15. Girou E, Brun-Buisson C, Taille S, Lemaire F, Brochard L. Secular trends in nosocomial infections and mortality associated with exacerbation of COPD and pulmonary edema. JAMA. 2003;290:2985-91.

16. Masip J, Roque M, Sánchez B, Fernández R, Subirana M, Expósito JA. Noninvasive ventilation in acute cardiogenic pulmo- nary edema: systematic review and meta-analysis. JAMA. 2005;294:3124-30.

17. Wysocki M, Tric L, Wolff MA, Millet H, Herman B. Noninvasive pressure support ventilation in patients with acute respiratory failure: a randomized comparison with conventional therapy. Chest. 1995;107:761-8.

18. Ferrer M, Esquinas A, Leon M, Gonzalez G, Alarcon A, Torres A. Non-invasive ventilation in severe hypoxemic respiratory failure. A randomized clinical trial. Am J Respir Crit Care Med. 2003; 168:1438-44.

19. Nava S, Carlucci A. Non-invasive pressure support ventilation in acute hypoxemic respiratry failure: common strategy for different pathologies? Intensive Care Med. 2002;28:1205-7.

20. Pennock BE, Kaplan PD, Carlin BW, Sabangan JS, Magovern JA. Pressure support ventilation with a simplified ventilatory support system administered with a nasal mask in patients with respiratory failure. Chest. 1991;100:1371-6.

21. Pennock BE, Crawshaw L, Kaplan PD. Noninvasive nasal mask ventilation for acute respiratory failure. Institution of a new therapeutic technology for routine use. Chest. 1994;105: 441-4.

22. Auriant I, Jallot A, Herve P, Cerrina J, Le Roy Ladurie F, Fournier JL, et al. Noninvasive ventilation reduces mortality in acute respiratory failure following lung resection. Am J Respir Crit Care Med. 2001;164:1231-5.

23. Keenan SP, Powers C, McCormack DG, Block G. Noninvasive positive-pressure ventilation for postextubation respiratory distress: a randomized controlled trial. JAMA. 2002;287:3238-44.

24. Esteban A, Frutos-Vivar F, Ferguson N, Arabi Y, Apezteguia C, Gonzalez M, et al. Noninvasive positive-pressure ventilation for respiratory failure after extubation. N Engl J Med. 2004;350:245260.

25. Girault C. Differences in success rates of noninvasive ventilation. JAMA. 2002;288:2540.

26. Meduri GU, Turner RE, Abou-Shala N, Wunderink R, Tolley E. Noninvasive positive pressure ventilation via face mask. Firstline intervention in patients with acute hypercapnic and hypoxemic respiratory failure. Chest. 1996;109:179-93.

27. Alsous F, Amoateng-Adjepong Y, Manthous CA. Noninvasive ventilation: experience at a community teaching hospital. Intensive Care Med. 1999;25:458-63.

28. Antonelli M, Conti G, Moro ML, Esquinas A, GonzalezDiaz G, Confalonieri M, et al. Predictors of failure of noninvasive positive pressure ventilation in patients with acute hypoxemic respiratory failure: a multi-center study. Intensive Care Med. 2001;27:1718-28.

29. Domenighetti G, Gayer R, Gentilini R. Noninvasive pressure support ventilation in non-COPD patients with acute cardiogenic pulmonary edema and severe community-acquired pneumonia: acute effects and outcome. Intensive Care Med. 2002;28:1226-32.

30. Demoule A, Girou E, Richard JC, Taille S, Brochard L. Benefits and risks of success or failure of noninvasive ventilation. Intensive Care Med. 2006;32;1756-65. 